\title{
Morfología de la semilla y anatomía de la cubierta seminal de cinco especies de Calliandra (Leguminosae-Mimosoideae) de Venezuela
}

Sirli Leython ${ }^{1} \&$ Damelis Jáuregui ${ }^{2}$

1. Fundación Instituto Botánico de Venezuela "Dr. Tobbias Lasser". Apdo. 2156,Caracas 1010-A, Venezuela. Fax +58212-6053970; sirli.leython@ucv.ve; sirlileython@yahoo.com

2. Instituto de Botánica Agrícola. Facultad de Agronomía. Universidad Central de Venezuela. Apdo. 4579. Maracay 2101. Edo. Aragua. Venezuela. Fax: +58-43-5507107; damelis.jauregui@agr.ucv.ve

Recibido 01-VI-2007. Corregido 30-VI-2008. Aceptado 31-VII-2008.

\begin{abstract}
Seed morphology and anatomy of the seed coat in five species of Calliandra (LeguminosaeMimosoideae) from Venezuela. Calliandra is an exclusively Neotropoical genus with 135 described species. There are 30 species in Venezuela. We studied seed morphology and the seed coat anatomy of five species: Calliandra riparia Pittier, Calliandra glomerulata Karsten var. glomerulata, Calliandra magdalenae (DC.) Benth. var. magdalenae, Calliandra surinamensis Benth. and Calliandra falcata Benth., to establish simililarities and differences amongst taxa for taxonomic characterization. Of the evaluated characters, 10 quantitative characters and 3 qualitative characters were selected as the most informative for the separation of species. Seed morphological characters of taxonomic value are form and size. Thickness of the light line, number of layers and space between the osteosclereids constitute distinctive characters. Rev. Biol. Trop. 56 (3): 1075-1086. Epub 2008 September 30.
\end{abstract}

Key words: Calliandra, leguminosae, mimosoideae, morphology, anatomy, seed coat.

Calliandra es un género exclusivamente neotropical con 135 especies (Barneby 1998, Souza y Queiroz 2004, Lewis et al. 2005). En Venezuela está representado por 30 especies, distribuidas principalmente en el sur del país. Crecen principalmente en bosques tropicales estacionalmente secos, ribereños, espinosos, arbustales, praderas enselvadas (cerrado) y matorral rocoso. Varias de las especies están adaptadas a ambientes desérticos (Barneby 1998, Lewis et al. 2005).

Algunas especies del género son ampliamente usadas como ornamentales, ejemplo de ello son C. falcata, C. purpurea, C. houstoniana, C. riparia, C. antioquiae, C. guildinguii y C. haematocephala, entre otras. En zonas muy áridas del oeste de Estados Unidos, C. eriophylla es aprovechada como forraje para ganado y venados. En medicina popular las raíces de algunas especies proveen productos para curar enfermedades oculares, diarrea e indigestión.
También en México se extraen taninos de la madera de $C$. houstoniana var. anomala (Izaquirre y Beyhaut 2003). Calliandra calothyrsus tiene un potencial agroforestal en Asia y África, donde fue introducida con este objetivo, y se plantea su incorporación en programas integrales agroforestales en su área de distribución natural (Hernández 1991).

Los caracteres morfológicos y anatómicos de las semillas de Mimosoideae en general, han sido considerados de importancia taxonómica, ya que permiten establecer similitudes y diferencias entre distintos taxones (Corner 1951, Gunn 1981, 1984, Escala 1999). Por su parte, Van Staden y Kelly (1989) señalaron, que la cubierta seminal muestra un patrón estructural constante en el grupo. En este sentido, se propone el presente trabajo, para caracterizar y comparar morfológicamente, así como describir anatómicamente la cubierta seminal de las semillas de cinco especies que crecen en la 
región andina de Venezuela, con el fin de determinar caracteres con posible valor taxonómico en el género Calliandra.

\section{MATERIALES Y MÉTODOS}

Para realizar el presente estudio se recolectaron frutos maduros de las especies Calliandra riparia Pittier, Calliandra glomerulata Karsten var. glomerulata, Calliandra magdalenae (DC.) Benth. var. magdalenae, Calliandra surinamensis Benth. y Calliandra falcata Benth. (Cuadro 1). Se extrajeron las semillas separándolas en dos lotes, uno para el estudio morfológico y otro para la caracterización anatómica, éstas últimas se fijaron en FAA (formaldehído, ácido acético, etanol 70\%).

Análisis morfológico: Para la caracterización morfológica se tomaron 15 semillas por especie, de diferentes individuos, de acuerdo a la disponibilidad en campo, consideradas características de todo el ámbito de variación morfológica de la especie y procedentes de localidades representativas de la distribución geográfica conocida en Venezuela para cada una. Para determinar el tamaño se midió largo, ancho y grosor ( $\mathrm{x}$ a $\mathrm{x} \mathrm{g}$ ) con papel milimetrado, siempre en este orden y expresado en milímetros. Los valores se refieren a promedios (Cuadro 2). El largo de las semillas fue medido desde el extremo hilar hasta el calazal; el ancho fue medido en la porción media y más ancha de la semilla al igual que el grosor (Fig. 1A). Para definir la forma de las semillas, se siguió lo indicado por Murley (1951) tomado de Bravato (1974) y Gunn (1984). El color fue estimado en semillas maduras. El pleurograma o línea fisural fue descrito siguiendo la terminología propuesta por Gunn (1984). El hilo y forma del lens se definió según Boelcke (1946), Bravato (1974), Gunn (1984) y Escala (1999). Se tomó un total de 15 medidas por especie para cada carácter.

Análisis anatómico: La cubierta seminal se separó manualmente en ocho semillas por especie, de diferentes individuos, teniendo en cuenta su disponibilidad en campo. Se tomaron porciones de la región media y del rafe de cada semilla y en cada región se realizaron 15 medidas por especie, los caracteres considerados para el análisis al igual que los valores promedios se observan en el Cuadro 2. Los cortes obtenidos se deshidrataron en una serie creciente de alcohol butílico terciario (TBA), y se imbibieron e incluyeron en parafina con punto de fusión $56^{\circ} \mathrm{C}$, siguiendo la metodología indicada por Johansen (1940). Después se seccionaron en un micrótomo de rotación, obteniéndose secciones transversales de $15 \mu \mathrm{m}$ de grosor. Las muestras obtenidas fueron teñidas con safranina-fastgreen (Johansen 1940) o con azul de toluidina acuosa (1\%) (Sakai
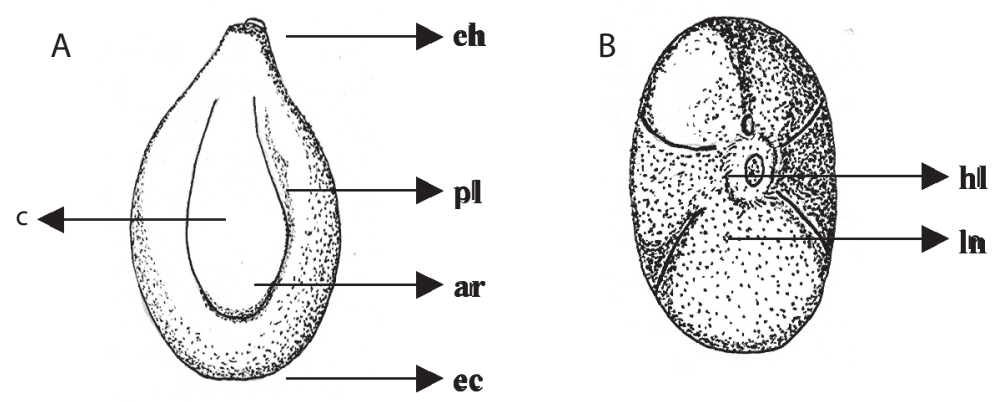

Fig. 1. Semilla de Calliandra con sus partes. (A.) Vista longitudinal. eh: extremo hilar, pl: pleurograma, c: centro, ar: areola, ec: extremo calazal. (B.) Vista frontal del extremo hilar. hl: hilo, ln: lens.

Fig 1. Seed parts in Calliandra. (A.) Longitudinal profile. eh: hilar extreme, pl: pleurogram, c: center, ar: areola, ec: calazal extreme. (B.) Frontal profile of the hilar extreme. hl: hilum, ln: lens. 
CUADRO 1

Especímenes de Calliandra de Venezuela utilizados para el estudio de semillas

TABLE 1

Specimens Venezuelan Calliandra studied

Especies
C. riparia

C. glomerulata var. glomerulata

C. magdalenae var. magdalenae

C. surinamensis

C. falcata
Ubicación

Estado Trujillo. 09²4’07” N, 7047’91” W. Matorral caducifolio

Estado Trujillo. 09³7’41” N, 70²5’30” W. Matorral caducifolio

Estado Táchira. $08^{\circ} 14^{\prime} 58^{\prime \prime} \mathrm{N}, 72^{\circ} 11^{\prime} 21^{\prime \prime}$ W. Bosque caducifolio.

Estado Táchira. $07^{\circ} 58^{\prime} 13^{\prime}$ N, $72^{\circ} 14$ '12” W. Bosque caducifolio.

Estado Mérida. Lagunillas, $08^{\circ} 17^{\prime} 47^{\prime \prime}$ N, $71^{\circ} 25^{\prime} 43^{\prime \prime} \mathrm{W}$. Cultivada en Jardín de Escuela.

Estado Táchira. $07^{\circ} 58^{\prime} 13^{\prime}$ N, $72^{\circ} 14^{\prime} 12^{\prime}$ W. Bosque caducifolio.

Estado Táchira. $07^{\circ} 58^{\prime} 13^{\prime \prime} \mathrm{N}, 72^{\circ} 14^{\prime} 12^{\prime}$ W. Bosque caducifolio.

Estado Táchira. $07^{\circ} 49^{\prime} 44^{\prime \prime} \mathrm{N}, 7^{\circ} 20{ }^{\prime} 37^{\prime \prime}$ W. Matorral caducifolio.

Estado Táchira. $07^{\circ} 48^{\prime} 19^{\prime \prime}$ N, 72²1'38” W. Bosque caducifolio.

Estado Táchira. $07^{\circ} 48^{\prime} 19^{\prime}$ N, 72²1'38” W. Bosque caducifolio.

Estado Táchira. $07^{\circ} 48^{\prime} 19^{\prime \prime} \mathrm{N}, 7^{\circ} 21$ '38” W. Bosque caducifolio.

Estado Táchira. $07^{\circ} 58^{\prime} 26^{\prime \prime} \mathrm{N}, 72^{\circ} 26^{\prime} 21^{\prime \prime} \mathrm{W}$. Matorral caducifolio

Estado Mérida, Lagunillas, Pueblo Viejo, 08²2’50” N, Leython, S. 602 (VEN) 71²9'43”W. Matorral caducifolio.

Estado Mérida. Cultivada en jardines de la ULA, Fac. Forestales. $8^{\circ} 45^{\prime} 83^{\prime \prime} \mathrm{N}, 71^{\circ} 10^{\prime} 95^{\prime \prime} \mathrm{W}$.
Especímenes y herbario

Leython, S. 564 (VEN)

Leython, S. 571 (VEN)

Leython, S. 585 (VEN)

Leython, S. 588 (VEN)

Leython, S. 603 (VEN)

Leython, S. 586 (VEN)

Leython, S. 587 (VEN)

Leython, S. 589 (VEN)

Leython, S. 591 (VEN)

Leython, S. 592 (VEN)

Leython, S. 593 (VEN)

Leython S. 597 (VEN)

Leython, S. 600 (VEN)
1973) en aquellas muestras donde la tinción con safranina-fastgreen no resultó. El montaje de láminas permanente se realizó en bálsamo de Canadá.

Con el fin de corroborar algunos caracteres observados se realizaron macerados, para lo cual se coloco trozos de cubierta seminal en solución de Jeffrey (Johansen 1940) durante una hora, a $60^{\circ} \mathrm{C}$. Posteriormente se lavó repetidas veces con agua destilada estéril y se tiñó con azul de toluidina en glicerina.

Se realizaron algunas pruebas histoquímicas en secciones obtenidas a mano alzada. Para detectar lignina se utilizó floroglucinol + ácido 
CUADRO 2

Caracteres morfológicos y anatómicos de la cubierta seminal de cinco especies de Calliandra de Venezuela

TABLE 2

Morphological and seed coat anatomical characters of five Calliandra species from Venezuela

\begin{tabular}{|c|c|c|c|c|c|}
\hline Caracteres & C. riparia & $\begin{array}{c}\text { C. glomerulata } \\
\text { var. glomerulata }\end{array}$ & $\begin{array}{c}\text { C. magdalenae } \\
\text { var. magdalenae }\end{array}$ & C. surinamensis & C. falcata \\
\hline Tamaño 1 x a x g (mm) & $8.5-6-1.5$ & $7-6.25-1.85$ & $9.5-6.5-3.5$ & $9.3-5-2$ & $4.75-7-2.1$ \\
\hline Funículo 1 (mm) & 0.2 & 0.5 & 0.2 & 0.4 & 0.2 \\
\hline Hilo 1 (mm) & 0.4 & 0.4 & 0.5 & $0.6-0.7$ & 0.7 \\
\hline Lens $1(\mathrm{~mm})$ & 0.1 & 0.2 & 0.5 & 0.8 & 0.4 \\
\hline Capa de Malpighi espesor $(\mu \mathrm{m})$ & 38.8 & 31.2 & 23 & 31.4 & 27.4 \\
\hline $\begin{array}{l}\text { Línea lúcida distancia desde } \\
\text { la parte más externa de las } \\
\text { macrosesclereidas }(\mu \mathrm{m})\end{array}$ & $\begin{array}{c}\text { RM:17.6 } \\
\text { RF: } 6.7\end{array}$ & $\begin{array}{c}\text { RM: } 12.8 \\
\text { RF: } 5.4\end{array}$ & $\begin{array}{l}\text { RM: } 7 \\
\text { RF: } 6.6\end{array}$ & $\begin{array}{c}\text { RM: } 8.0 \\
\text { RF: ausente }\end{array}$ & $\begin{array}{l}\text { RM: } 12.1 \\
\text { RF: } 5.0\end{array}$ \\
\hline Hipodermis espesor $(\mu \mathrm{m})$ & $\begin{array}{l}\text { RM: } 17.4 \\
\text { RF: } 21.4\end{array}$ & $\begin{array}{l}\text { RM: } 10.4 \\
\text { RF: } 22.4\end{array}$ & $\begin{array}{l}\text { RM: } 12.5 \\
\text { RF: ausente }\end{array}$ & $\begin{array}{l}\text { RM: } 11.0 \\
\text { RF: } 75.8\end{array}$ & $\begin{array}{l}\text { RM: } 13.4 \\
\text { RF:ausente }\end{array}$ \\
\hline Células obliteradas espesor $(\mu \mathrm{m})$ & RM: 47.2 & RM: 51.8 & RM: 57.5 & RM: 56.0 & RM: 47.5 \\
\hline $\begin{array}{l}\text { Células redondeadas } \\
\text { espesor }(\mu \mathrm{m})\end{array}$ & $\begin{array}{c}\text { RM: } 89.6 \\
\text { RF: } 90\end{array}$ & $\begin{array}{l}\text { RM: ausente } \\
\text { RF: } 56\end{array}$ & $\begin{array}{l}\text { RM: } 88 \\
\text { RF: } 88\end{array}$ & $\begin{array}{l}\text { RM: } 21.8 \\
\text { RF: ausente }\end{array}$ & $\begin{array}{c}\text { RM:ausente } \\
\text { RF: } 77.2\end{array}$ \\
\hline
\end{tabular}

$1 \mathrm{x}$ a $\mathrm{x}$ g: largo $\mathrm{x}$ ancho $\mathrm{x}$ grosor

RM: región media RF: región del rafe

RM: middle region RF: region of raphe

clorhídrico (Roth 1964); la identificación de grasa se realizó usando Sudán III (Johansen 1940) y la determinación de taninos con cloruro férrico (Werker et al. 1979). Todas las secciones fueron montadas en agua-glicerina (1:1).

Las láminas obtenidas fueron observadas, analizadas y fotografiadas en un microscopio óptico.

Análisis estadísticos: el tratamiento estadístico consistió en aplicar la Función de Análisis Discriminante (Siegel 1956), con uso del programa de cálculo estadístico STATISTICA versión 5.5, para determinar las variables (caracteres) discriminantes entre las especies estudiadas (hipótesis nula).

\section{RESULTADOS \\ Calliandra riparia}

Características morfológicas: Cuatro a seis semillas por fruto, ovaladas; caras convexas; color castaño claro; superficie lisa, opaca; pleurograma color blanco, abarcando el $90 \%$ de la superficie de la semilla, brazos de igual longitud y areola grande (Fig. 2A); extremo hilar y calazal agudo; funículo color crema; hilo elíptico, con restos de funículo, apical; lens elíptico, prominente, castaño oscuro (Cuadro 2).

Características anatómicas: se distingue externamente la capa de Malpighi con línea 

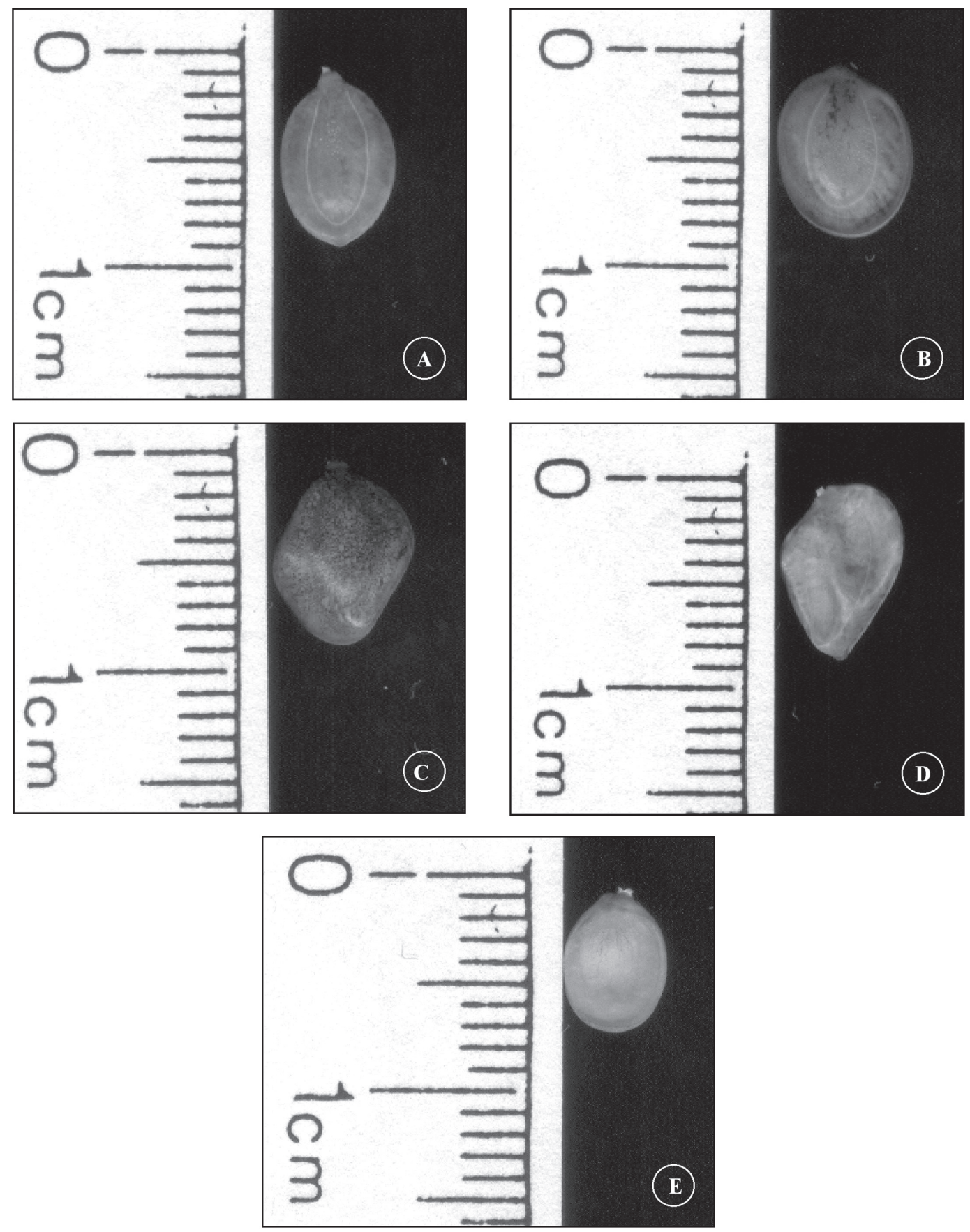

Fig. 2. Semillas de cinco especies de Calliandra de Venezuela, nótese sus características morfológicas. Detalles de la vista longitudinal. (A.) C. riparia. (B.) C. glomerulata var. glomerulata. (C.) C. magdalenae var. magdalenae. (D.) C. surinamensis. (E.) C. falcata.

Fig. 2. Seeds of five Calliandra species from Venezuela; observe morphological features. Detail of longitudinal profile. (A.) $C$. riparia. (B.) C. glomerulata var. glomerulata. (C.) C. magdalenae var. magdalenae. (D.) C. surinamensis. (E.) C. falcata. 
lúcida ubicada en la parte más externa de las macroesclereidas que conforman esta capa. Seguidamente, la hipodermis, uniestratificada en la región media con espacios intercelulares relativamente amplios (Fig. 3A), mientras que en el rafe está constituida por tres a cuatro estratos y espacios intercelulares relativamente pequeños. Internamente en la zona media son evidentes cinco estratos de células obliteradas con paredes engrosadas (Fig. 3A); seguidas de dos hileras de células redondeadas con paredes delgadas; en el rafe se presentan cuatro a cinco capas de células redondeadas con paredes gruesas (Cuadro 2).

\section{Calliandra glomerulata var. glomerulata}

Características morfológicas: Tres a seis semillas por fruto; orbiculares; caras convexas; color castaño claro con manchas castaño oscuro esparcidas en la superficie de la semilla; superficie lisa, opaca; pleurograma color crema, abarcando el $90 \%$ de la superficie de la semilla, brazos de igual longitud casi unidos al extremo hilar; areola grande; extremo hilar obtuso; extremo calazal redondeado (Fig. 2B); funículo color crema; hilo elíptico, subapical; lens orbicular, castaño oscuro con línea blanca central (Cuadro 2).

Características anatómicas: Externamente se distingue la capa de Malpighi en la región media con línea lúcida ubicada en la parte más externa de las macroesclereidas que conforman esta capa, tanto en la región media como en la del rafe. Seguidamente la hipodermis uniestratificada en la región media, formada por osteosclereidas con espacios intercelulares relativamente pequeños (Fig. 3B), mientras que en el rafe está formada por tres estratos y espacios intercelulares comparativamente pequeños. Internamente en la región media se observan siete a ocho hileras de células obliteradas, generalmente de paredes engrosadas (Fig. 3B); en el rafe seis a siete hileras de células redondeadas con paredes gruesas (Cuadro 2).

\section{Calliandra magdalenae var. magdalenae}

Características morfológicas: Cuatro a seis semillas por fruto, ovaladas a anchamente ovadas; caras convexas; color castaño oscuro, con manchas castaño oscuro esparcidas en la superficie de la semilla; superficie lisa, brillante; pleurograma color crema, abarcando el $90 \%$ de la superficie de la semilla, brazos de igual longitud unidos al extremo hilar; areola grande; extremo hilar redondeado; extremo calazal redondeado (Fig. 2C); funículo corto, color blanco; hilo elíptico, con restos de funículo, subapical; lens obovoide, prominente, castaño claro (Cuadro 2).

Características anatómicas: Externamente se distingue la capa de Malpighi (Fig. 3C) con línea lúcida ubicada en la parte más externa de las macroesclereidas que conforman dicha capa tanto en la región media (Fig. 3C) como en el rafe. Inmediatamente la hipodermis uniestratificada en la región media y espacios intercelulares relativamente pequeños (Fig. 3C), ausente en el rafe. Internamente en la región media se observan cinco a seis estratos de células obliteradas (Fig. 3C); seguidas de tres hileras de células redondeadas con paredes delgadas; en el rafe siete a nueve estratos de células redondeadas con paredes gruesas (Cuadro 2).

\section{Calliandra surinamensis}

Características morfológicas: Tres a siete semillas por fruto, ovaladas a anchamente ovaladas; caras convexas; color castaño claro con manchas castaño oscuro esparcidas en la superficie de la semilla; superficie lisa, brillante; pleurograma color blanco abarcando el $90 \%$ de la superficie de la semilla, brazos de igual longitud casi unidos al extremo hilar; extremo hilar obtuso a puntiforme; extremo calazal puntiforme (Fig. 2D); funículo color crema; hilo elíptico con restos de funículo, apical o subapical; línea central prominente produciendo engrosamiento en la región media, 

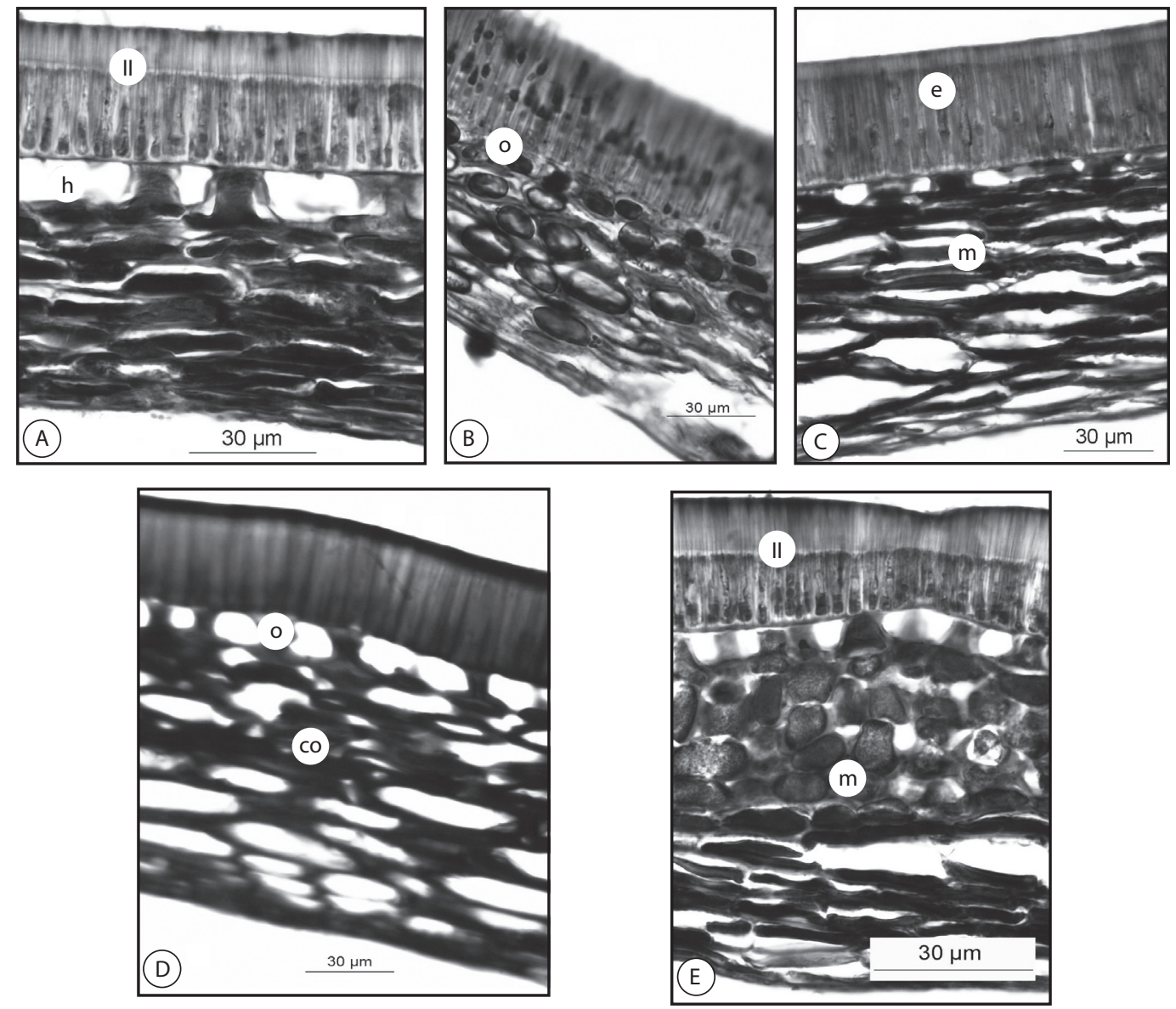

Fig. 3. Secciones transversales de la cubierta seminal en la región media, de cinco especies de Calliandra de Venezuela. (A.) C. riparia, nótese la línea lúcida (1l) y la hipodermis (h) con grandes espacios intercelulares. (B.) C. glomerulata var. glomerulata, vista de la capa de Malpighi (e), detalle del mesofilo (m) multiestratificado. (C.) C. magdalenae var. magdalenae, osteosclereidas (o) que constituyen la hipodermis. (D.) C. surinamensis, osteosclereidas (o) que conforman la hipodermis y células obliteradas (co) con grandes espacios intercelulares. (E.) C. falcata, detalle de la línea lúcida (11), vista del mesofilo (m).

Fig. 3. Cross-sectional sections in the seed coat middle region of five species of Calliandra from Venezuela. (A.) $C$. riparia, detail of light line (11), hypodermis (h) with great intercellular spaces. (B.) C. glomerulata var. glomerulata, detail of Malpighi layer (cM) (e), detail of mesophyll (m) multistratified. (C.) C. magdalenae var magdalenae, osteosclereids (o) from the hypodermis. (D.) C. surinamensis, osteosclereids (o) from the hypodermis, obliterated cells (co) with great intercellular spaces. (E.) C. falcata, detail of light line (1l), detail of mesophyll (m). 
lens orbicular, prominente, castaño oscuro, considerado grande (Cuadro 2).

Características anatómicas: externamente se diferencia la capa de Malpighi en la región media (Fig. 3D) con línea lúcida situada en la parte más externa de las macroesclereidas que constituyen dicha capa (Fig. 3D), ausente en el rafe. Seguidamente la hipodermis uniestratificada en la región media con espacios intercelulares relativamente amplios (Fig. 3D); y en el rafe está constituida por cinco a seis estratos. Internamente en la región media cinco a siete estratos de células obliteradas, generalmente de paredes engrosadas (Fig. 3D), continuadas por dos a tres estratos de células redondeadas con paredes delgadas (Cuadro 2).

\section{Calliandra falcata}

Características morfológicas: Tres a cuatro semillas por fruto, elípticas; caras aplanadas; color castaño claro; superficie rugosa, brillante; pleurograma color blanco tenue, abarcando el $90 \%$ de la superficie de la semilla, brazos de igual longitud casi unidos y prominentes hacia el extremo hilar, ligeramente evidente hacia el extremo calazal; areola grande con líneas de fractura; extremo hilar agudo; extremo calazal redondeado (Fig. 2E); funículo de grosor uniforme, color crema; hilo elíptico, con restos de funículo, apical; lens elíptico, prominente, castaño oscuro (Cuadro 2).

Características anatómicas: Externamente se distingue la capa de Malpighi (Fig. $3 \mathrm{E}$ ), con línea lúcida situada en la parte más externa de las macroesclereidas que constituyen la mencionada capa (Fig. 3E); tanto en la región media como en la del rafe. Inmediatamente la hipodermis de cuatro a cinco estratos en la región media (Fig. 3E), espesor y espacios intercelulares relativamente grandes (Fig. 3E); ausente en el rafe. Internamente en la zona media son evidentes seis a siete estratos de células obliteradas (Fig. 3E); en el rafe ocho estratos de células redondeadas con paredes gruesas (Cuadro 2).
Tomando en consideración la forma de las semillas, las especies tienen predominantemente forma ovalada, ya que se observa en tres de las cinco especies estudiadas: $C$. riparia, $C$. magdalenae var. magdalenae y C. surinamensis. En función de valores absolutos, $52 \%$ de los individuos presentaron forma ovalada, $20 \%$ forma orbicular y elíptica y $8 \%$ forma anchamente ovada. En cuanto al color la tendencia general observada fue castaño.

La función de análisis discriminante (Cuadro 3) indica que estadísticamente se observa un comportamiento bastante heterogéneo entre las especies estudiadas, es decir existe diferencias significativas $(p<0,0000)$ que facilita la separación taxonómica entre las especies estudiadas, donde el tamaño del lens fue el carácter con más poder discriminante (p-level 0,000000) y la línea lúcida (distancia desde la parte más externa de las macrosesclereidas) el de menor poder discriminante (p-level 0,005789). Se formaron cinco grupos homogéneos con superposición entre sí.

Características histoquímicas de la cubierta seminal: El Cuadro 4 muestra los resultados de las pruebas realizadas. Se determinó que existe grasa en todas las especies estudiadas al observarse una coloración naranja a nivel de la cutícula e inclusive en el interior de la capa de Malpighi (Fig. 4A). Se detectaron taninos en la mayoría de las capas, excepto en la capa de Malpighi al observarse una coloración oscura (Fig. 4B). No se observó lignina en las especies en estudio.

\section{DISCUSIÓN}

La presente investigación ha demostrado que la morfología de la semilla y la anatomía de la cubierta seminal de las especies de Calliandra, aportan información de significación taxonómica.

El estudio morfológico evidencia amplia variabilidad, por lo que no hay dificultad para distinguir las especies. Así, con relación a la forma, tamaño y color de las semillas se pudo determinar que poseen un polimorfismo 


\section{CUADRO 3}

Resultados de la Función de Análisis Discriminante

TABLE 3

Results of the Discriminant Analysis

Análisis Discriminante N: 75

Variables

Largo del Lens

Capa Malpighi espesor

Células obliteradas espesor

Largo del Funículo

Ancho de la semilla

Largo de la semilla

Largo del Hilo (de la semilla)

Hipodermis espesor

Grosor de la semilla

Línea lúcida distancia desde la parte más externa de las macrosesclereidas
$\mathrm{N}^{\mathrm{o}}$ de variables en el modelo: 10 , Grupos: 5 (especies); Wilk's Lambda: 0,00005 aprox; F $(40,229)=72,811 p<0,0000$

Wilk's Lambda

F (g.l)

p-level

0.000084

32.33 (4. 69)

0.000000

0.000086

11.30 (4. 68)

0.000001

0.000077

8.46 (4. 67)

0.000018

0.000082

10.13 (4. 66)

0.000002

0.000071

$6.76(4.65)$

0.000147

0.000102

16.19 (4. 64)

0.000000

0.000070

6.33 (4. 63)

0.000258

0.000066

$5.30(4.62)$

0.001014

0.000067

5.42 (4. 61)

0.000860

0.000062

4.04 (4. 60)

0.005789

\section{CUADRO 4}

Características histoquímicas de la cubierta seminal de cinco especies de Calliandra de Venezuela

\section{TABLE 4}

Histochemical characteristics of the seed coat of five Calliandra species from Venezuela

$\begin{array}{lccc}\text { Especies } & \text { Lignina } & \text { Grasa } & \text { Taninos } \\ \text { C. riparia } & - & + & + \\ \text { C. glomerulata var. glomerulata } & - & + & + \\ \text { C. magdalenae var. magdalenae } & - & + & + \\ \text { C. } \text { surinamensis } & - & + & + \\ \text { C. falcata } & - & + & +\end{array}$

Presencia $(+) /$ Ausencia $(-)$

Presence (+) / Absence (-) 

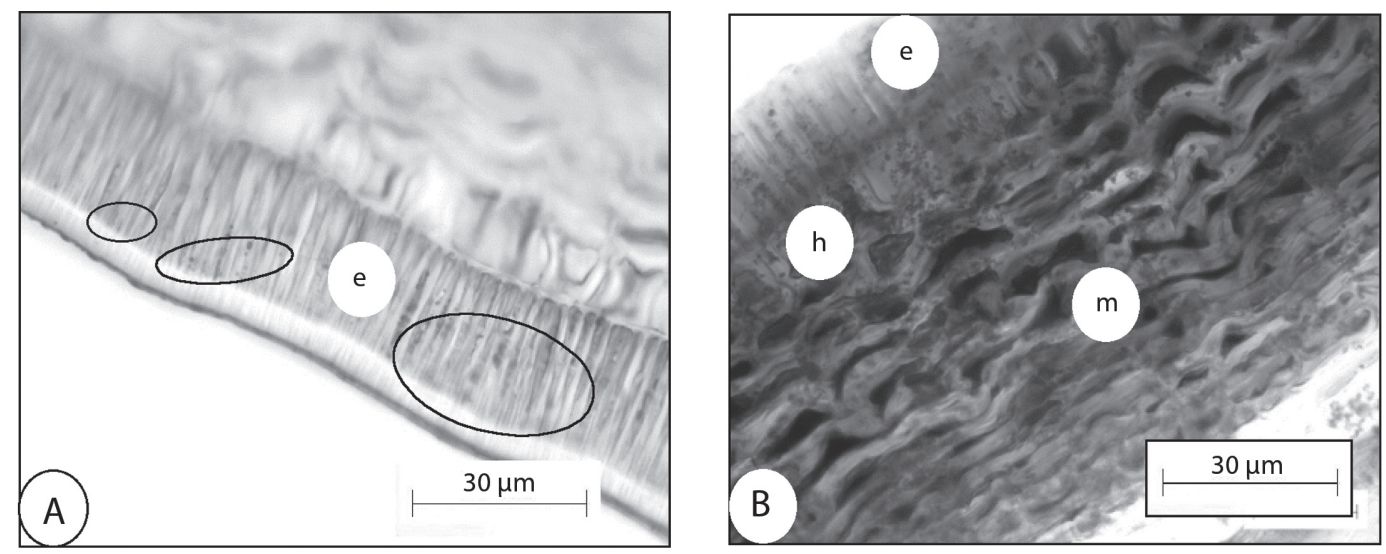

Fig. 4. Secciones transversales de la cubierta seminal mostrando reacción a dos pruebas histoquímicas. (A.) C. falcata, nótese la presencia de grasa en la capa de Malpighi (e) y en cutícula. (B.) C. falcata, obsérvese la presencia de taninos en la capa de Malpighi (cM), hipodermis (h) y mesofilo (m).

Fig. 4. Cross-sectional sections of the seed coat showing reaction to histochemical tests. (A.) C. falcata, observe the lipids in the Malpighi layer (e). (B.) C. falcata, observe the tannin in the Malpighi layer (cM), hypodermis (h) and mesophyll (m).

marcado. Estos resultados confirman los obtenidos por (Bravato 1974).

De acuerdo con Corner (1976) y Gunn (1981, 1984) el pleurograma, contribuye taxonómicamente en la determinación específica, ellos señalan que el mismo se encuentra en un 65 a $70 \%$ de los géneros de Mimosoideae, y que es de gran importancia el área que ocupa en la semilla y la longitud de los brazos. En las especies estudiadas se observó el pleurograma, siendo los brazos de igual longitud.

Los resultados difieren de lo señalado por Escala (1999) respecto al hilo, quien indica que esta característica es de poco valor diagnóstico en la subfamilia. De acuerdo a lo obtenido, se propone que el tamaño y forma del hilo pueden ser otras características morfológicas de utilidad para ser consideradas en la valoración sistemática del género Calliandra. Asimismo, el tamaño, la forma y el color del lens revisten gran importancia y pueden considerarse de valor diagnóstico para distinguir estas cinco especies de Calliandra.

La anatomía de la cubierta seminal mantuvo el patrón constante indicado para la subfamilia (Corner 1951, 1976, Gunn 1981), caracterizado por la capa de Malpighi, hipodermis formada por osteosclereidas y parénquima colapsado. Sin embargo, existen variaciones cuantitativas en el espesor de estas regiones que permiten distinguir las especies (Cuadro 2 y 3 ).

Los análisis estadísticos indican que existen diferencias estadísticamente significativas $(p=0,000001)$ en el espesor de la capa de Malpighi. Calliandra riparia registra el valor promedio más alto y se diferencia de las restantes especies en estudio. Por su parte C. magdalenae var. magdalenae y C. falcata muestran los valores promedios más bajos en el espesor de esta capa, sin diferencias estadísticas entre ambas, pero sí con relación a las otras especies en estudio.

Otro carácter de la anatomía de semillas considerado de valor diagnóstico en las especies estudiadas es la línea lúcida, ya que su posición varía en la capa de macroesclereidas que conforman la capa de Malpighi. Escala (1999) considera que la línea lúcida es un carácter constante en las especies de Mimosoideae; sin embargo, Corner $(1951,1976)$ indica que muchas especies de Leguminosae no tienen línea lúcida, excepto en la zona del hilo. Los resultados indican que existen diferencias estadísticamente significativas $(\mathrm{p}=0,005789)$, por 
lo que esta variable contribuye en gran medida a la separación de los cinco grupos (especies) formados en el análisis discriminante (Cuadro 3). En C. riparia y C. falcata se evidencia casi en la porción media de las macroesclereidas, mientras que en las otras especies se ubica más externamente.

El espesor de la hipodermis, tanto en la región media como en el rafe contribuyen a distinguir las especies analizadas (Cuadro 3). Calliandra riparia muestra el valor promedio más alto de espesor en la región media, mientras que en la región del rafe, el valor promedio más alto lo tiene Calliandra surinamensis (Cuadro 2).

El parénquima de las semillas está constituido por células obliteradas y células redondeadas, con diferencias estadísticamente significativas en su espesor $(p=0,000018)$, esta variable resultó diferente en todas las especies, tanto en la región media como en el rafe, por lo que podría ser empleada también para distinguir las especies estudiadas.

En general se puede indicar que el arreglo histológico observado es característico de semillas de Leguminosae, sin embargo, las variaciones en espesor de las diferentes capas pudiesen estar asociadas con diferencias en la capacidad de las semillas para germinar, lo cual tiene importancia adaptativa, por cuanto promueve la supervivencia de las plantas en condiciones adversas. La presencia de una epidermis conformada por macroesclereidas en empalizada, muy compacta, línea lucida, osteoesclereidas y el alto contenido de compuestos hidrofóbicos como taninos, pudiesen determinar impermeabilidad en las semillas, tal como se ha referido para otros taxones de Leguminosae (Werker et al. 1979, SerratoValenti et al. 1994, 1995).

La literatura no hace mención a la composición química de la cubierta seminal en especies de Calliandra, por lo que se puede considerar el presente estudio como un primer aporte. El contenido de taninos observado podría actuar como mecanismo de protección y regulación de la permeabilidad, se ha indicado que la acumulación de compuestos fenólicos es ventajosa, debido a que pueden ligarse a polisacáridos de las paredes celulares incrementando $\mathrm{su}$ rigidez y resistencia a la biodegradación y por otra parte pueden ser tóxicos en grados variables a diferentes predatores (Werker et al. 1979). Asimismo la acumulación de grasas pudiese asociarse con impermeabilización de semillas y quizás con un reservorio energético para el desarrollo posterior del embrión. Las especies estudiadas habitan en bosques caducifolios y matorrales caducifolios, sometidos a un período de sequía durante el año, por lo que las semillas deben presentar algún mecanismo de latencia que asegure su supervivencia, al presente no se ha identificado si lo presentan y si ésta es física.

Estos resultados se refieren únicamente a las especies estudiadas; quedan por considerar las demás especies de Calliandra, que sería interesante analizar para tratar de establecer el valor de los caracteres diferenciales analizados.

\section{RESUMEN}

Se estudió la morfología de las semillas y la anatomía de la cubierta seminal en cinco especies venezolanas del género Calliandra, con la finalidad de establecer similitudes y diferencias entre estos taxa, para su utilización como criterio taxonómico. Se analizaron caracteres morfológicos de valor taxonómico; entre ellos la forma y el tamaño de la semilla, así como anatómicos tales como espesor de las diferentes regiones, la ubicación de la línea lúcida y el tamaño relativo de los espacios intercelulares en la hipodermis. Con los resultados se realizaron análisis de agrupamiento por medio de la función de análisis discriminante. Diez caracteres cuantitativos, y tres caracteres cualitativos, resultaron informativos para la separación de especies.

Palabras clave: Calliandra, Leguminosae, Mimosoideae, morfología, anatomía, semilla, cubierta seminal

\section{REFERENCIAS}

Barneby, R. 1998. Calliandra. Silk tree, Guanacaste, Monkey`s Earring. A generic system for the Synandrous Mimosaceae of the Americas. Mem. New York Bot. Gard. 74: 1-223.

Boelcke, O. 1946. Estudio morfológico de las semillas de Leguminosas Mimosoideas y Caesalpinioideas de interés agronómico en la Argentina. Darwiniana. 7: 240-325. 
Bravato, M. 1974. Estudio morfológico de frutos y semillas de las Mimosoideae (Leguminosae) de Venezuela. Acta Bot. Venez. 9: 317-361.

Corner, E. 1951.The Leguminous seed. Phytomorphology 1: $117-150$.

Corner, E. 1976. The seeds of Dicotyledons. Vol. I. Cambridge University. Madingley Road, Cambridge, England. 309 p.

Escala, M. 1999. Estudio morfoanatómico de frutos y semillas de Leguminosas de los Altos Llanos Centrales de Venezuela (Estación Biológica de los Llanos de La Sociedad Venezolana de Ciencias Naturales). Bol. Soc. Venez. Ci. Nat. 148: 259-316.

Gunn, C. 1981. Seeds of Leguminosae. In Polhill, R. \& H. Raven (eds.). Advances in Legume Systematics. Royal Botanic Garden. Kew, London, England. 2: 913-925.

Gunn, C. 1984. Fruits and seeds of Genera in the Subfamily Mimosoideae (Fabaceae). Agric. Res. Serv. Technical Bulletin. U.S.D.A. 1681. 194 p.

Hernández, H. 1991. Taxonomía, distribución geográfica y biología reproductiva de Calliandra calothyrsus (Leguminosae, Mimosoideae), una especie con potencial agroforestal. Anales Inst. Biol. Univ. Nac. Auton. México, Bot. 62: 121-132.

Johansen, D. 1940. Plant microtechnique. McGrawHill. Newark, New York, USA. 45 p.

Izaguirre, P. y R. Beyhaut. 2003. Las Leguminosas en Uruguay y regiones vecinas. Parte 2 y 3 . Hemisferio Sur. Tacuarembó, Montevideo, Uruguay. 302 p.
Lewis, G., B. Schride, B. Mackinder \& M. Lock (eds.). 2005. Legumes of the World. Royal Botanic Gardens, Kew, London, England. 577 p.

Roth, I. 1964. Microtecnia vegetal. Universidad Central de Venezuela. Caracas. Venezuela. 34 p.

Sakai,W. 1973. Simple metod for differential staining of paraffin embedded plan material using toluidina blue O. Stain Technology. Baltimore, Maryland, USA. $124 \mathrm{p}$.

Serrato-Valenti, G. L. Cornara, P. Ghisellini \& M. Ferrando. 1994. Testa estructure and histochemistry related to water uptake in Leucaena leucocephala Lam (De Wit). Ann. Bot. 73: 513-537.

Serrato-Valenti, G. M. De Vries \& L. Cornara. 1995. The region hilar in Leucaena leucocephala Lam (De Wit) seed: structure, histochemistry and the role of lens in germination. Ann. Bot. 73: 569-574.

Siegel, S. 1956. Nonparametric statistics for the behavioral sciences. McGrawHill. Newark, New York, USA. $313 \mathrm{p}$.

Souza, E. \& L. Queiroz. 2004. Duas novas espécies de Calliandra Benth. (Leguminosae - Mimosoideae) da Chapada Diamantina, Bahia, Brasil. Rev Bras Bot. 27: 615-619.

Van Staden, J., \& K. Nelly. 1989. Legume seeds-The structure: function equation. In Stirton. C. \& J. Zarucchi (eds.). Advances in Legume Biology. Monogr. Syst. Bot. Missouri Bot. Gard. 29:417-450.

Werker, E., I. Marbach \& A. Mayer. 1979. Relation between the anatomy of the testa, water permeability and the presence of phenolics in the genus Pisum. Ann. Bot. 43: 765-771. 